\title{
Ottobre Partigiano: esperimenti di Public History per raccontare la Resistenza a Parma
}

L'articolo propone un resoconto della rassegna organizzata dall'Isrec di Parma nell'ottobre 2019 dedicata alla Resistenza. Sviluppata in quattro incontri, aperti alla cittadinanza, sono stati affrontati diversi temi su questa stagione storica attraverso modalità e linguaggi espositivi diversi: letture sceniche, public history, letteratura, film. Ogni serata è stata proposta con un suo accompagnamento musicale.

This article is a report of a conference organized by Isrec Parma on October 2019 in order to deepen the theme of the Resistance. The idea is to dedicate a series of four meetings, open to citizenship, to deepen knowledge about the historical period 1943-1945, through different methods and exhibition languages, like stage readings, public history, literature and movies. Each meeting was proposed with its own musical accompaniment.

Nel mese di ottobre 2019 l'Istituto storico della Resistenza e dell'età contemporanea di Parma (Isrec) ha organizzato la prima edizione della rassegna Ottobre Partigiano costituita da quattro incontri aperti alla cittadinanza.

L'obiettivo degli organizzatori, Isrec in collaborazione con Regione Emilia-Romagna e Comune di Parma, è quello di dedicare una settimana di eventi e di riflessioni, al di fuori dei momenti prettamente celebrativi che accompagnano la ricorrenza del 25 aprile, gettando lo sguardo agli eventi del 1943-45 attraverso linguaggi differenti, letteratura, arti visive, teatrali, musicali e di divulgazione storica coinvolgendo protagonisti di diverse discipline culturali.

Si è iniziato il 25 ottobre presso l'auditorium della Casa della musica dove si è tenuto il primo incontro: Raccontare per la storia. Ines e Luisa partigiane che ha approfondito le vicende di due donne, Ines Bedeschi e Luisa Calzetta, attive 


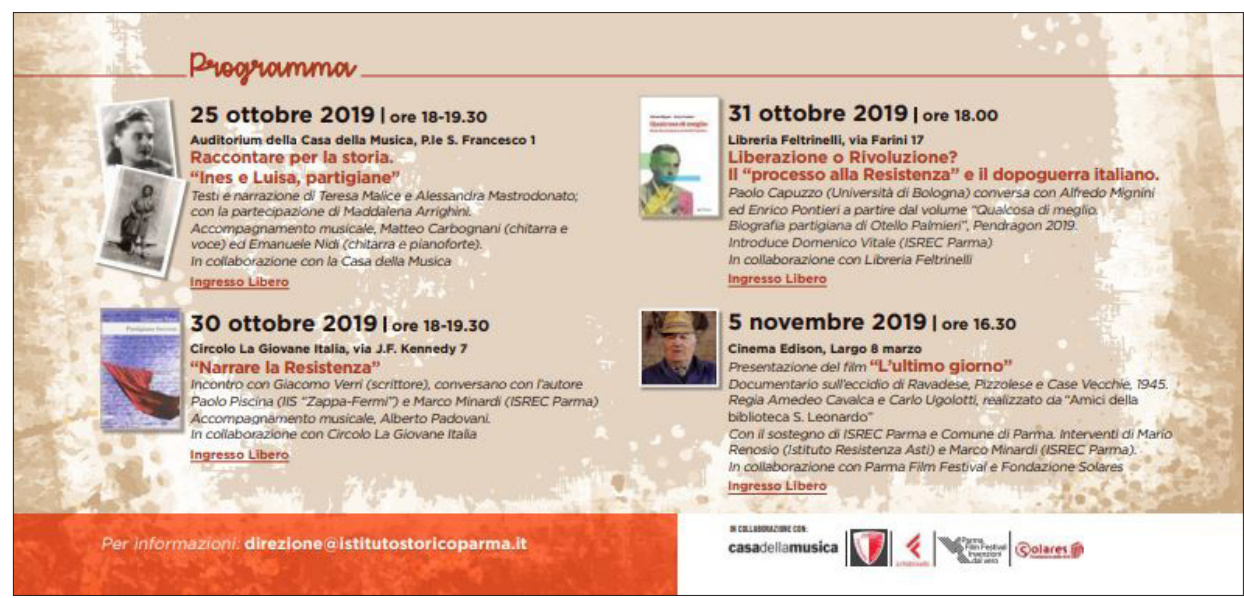

Fig. 1. Locandina

nel Parmense e rimaste uccise nella lotta di Liberazione. Ines Bedeschi (19141945), la partigiana Bruna, è originaria del Ravennate e appartiene a una famiglia socialista che per tutto il Ventennio subisce le violenze squadriste. Si sposa molto giovane con un coetaneo che però si rivela un fervente fascista e, anche per questa incompatibilità di convinzioni politiche, Ines decide di lasciarlo e di tornare alla casa paterna, non senza dare scandalo per questa scelta. Rientrata in famiglia si lascia coinvolgere nella lotta al fascismo, collaborando come staffetta per il Comando unico militare Emilia-Romagna (Cumer) e il partito comunista clandestino, e dal Ravennate viene trasferita nel Parmense come collegamento tra i vari gruppi partigiani. Arrestata a Parma nel gennaio 1945 e tenuta in carcere per un mese, durante il quale subisce torture, viene uccisa a marzo tramite fucilazione insieme ad altri due compagni sulle rive del Po e il suo corpo non verrà mai ritrovato.

Fig. 2. Lettura scenica

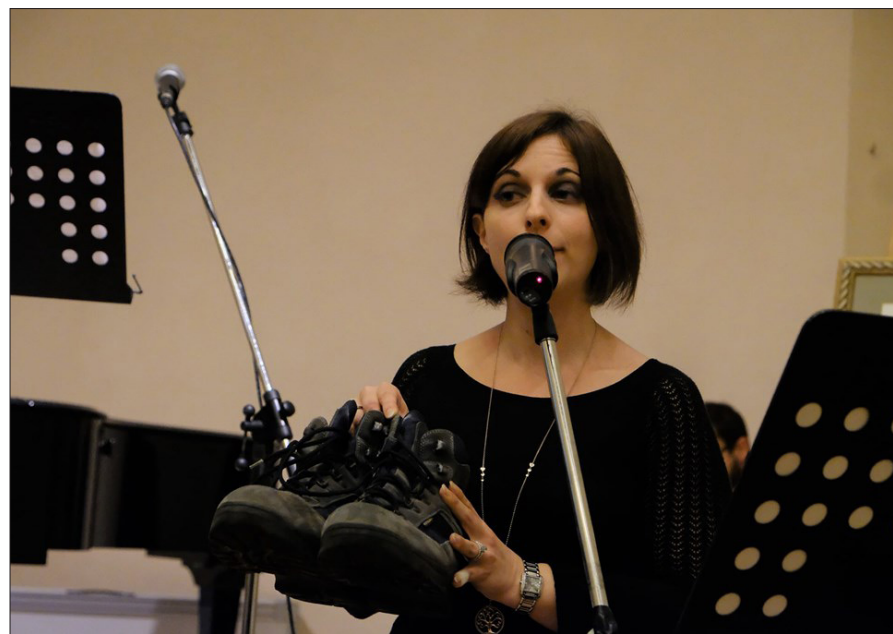


Luisa Calzetta (1909-1944) nasce a New York da emigrati dell'Appennino parmense e fa ritorno in Italia nel 1928, in pieno regime fascista. Studia per diventare maestra elementare crescendo in una famiglia convintamente antifascista. Dopo l'8 settembre del 1943 la sua casa di Compiano diventa rifugio per molti soldati sbandati e per i primi aderenti ai gruppi partigiani. Lei stessa dall'estate del 1944 decide di entrare in una brigata, la $61^{\text {a }}$ brigata Giuseppe Mazzini operativa in Val Nure, con il nome di battaglia Tigrona e partecipa a diverse azioni armate per liberare la valle.

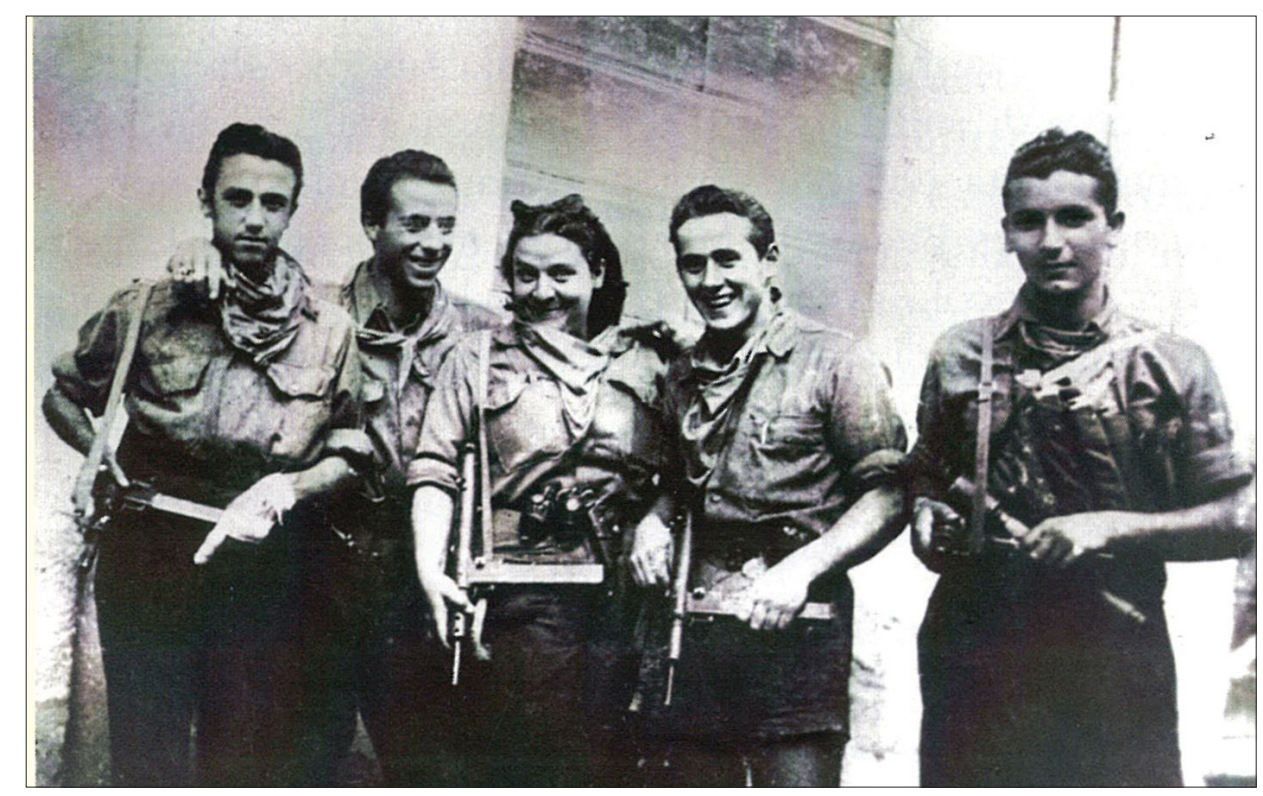

Fig. 3. Luisa Calzetta "Tigrona" e i suoi compagni di Brigata

Muore durante uno scontro a fuoco con i nazifascisti in montagna nel dicembre 1944 al passo dei Guselli, tra la Val Nure e la Val d'Arda, nel corso di un attacco militare che colpisce tutto l'Appennino piacentino e parmense. Entrambe le donne alla fine della guerra verranno insignite della Medaglia al valor militare alla memoria per il proprio sacrificio. La lettura scenica, intervallata da momenti di musica curati da Emanuele Nidi (pianoforte e voce) e Matteo Carbognani (chitarra e voce), ha visto dialogare tra loro le studiose Teresa Malice e Alessandra Mastrodonato, che hanno presentato testi e riflessioni suscitati dalle esperienze delle due partigiane. Maddalena Arrighini, oltre a collaborare alla lettura, ha presentato un approfondimento sulla presenza delle prigioniere politiche negli istituti penitenziari della Repubblica sociale italiana. Una serata, quindi, dedicata a mettere in luce l'importanza del contributo femminile, espresso in diverse forme, 
al fenomeno della Resistenza, che ha permesso una volta terminato il conflitto di fare grandi passi avanti sul piano dei diritti concessi alle italiane, a cominciare dall'accesso al voto nel 1946.

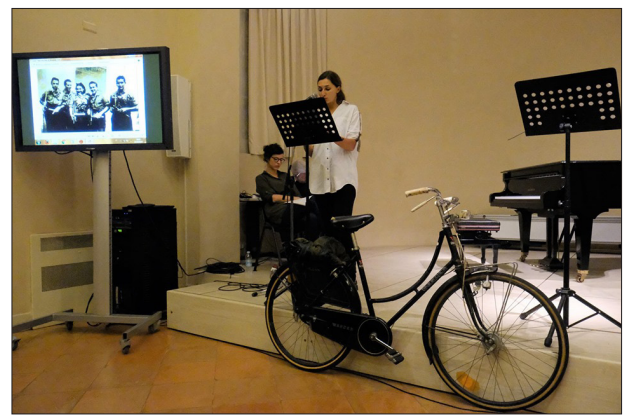

Fig. 4. Lettura scenica

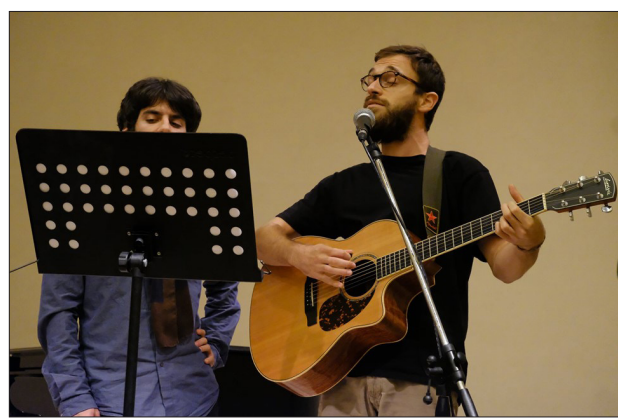

Fig. 5. Accompagnamento musicale

Il secondo appuntamento della rassegna si è tenuto mercoledì 30 ottobre presso il circolo Giovane Italia e ha affrontato il tema: Narrare la Resistenza. Paolo Piscina (Istituto di istruzione superiore Zappa-Fermi, Borgo Val di Taro) e lo storico Marco Minardi (Isrec Parma) hanno conversato con Giacomo Verri, scrittore ma anche insegnante a Borgosesia, dove è nato 42 anni fa: autore dei romanzi Racconti partigiani [Verri 2015], Partigiano Inverno [Verri 2012], Un altro candore [Verri 2019] e finalista al Premio Italo Calvino nel 2011. La serata è stata intermezzata da un accompagnamento musicale a cura di Alberto Padovani. Il dialogo si è sviluppato su un doppio binario: da un lato, la Resistenza quale tema virtuoso e ricco di suggestioni colte dalla letteratura e dalla scrittura creativa e poetica, con linguaggi originali e libertà narrativa; e dall'altro, l'inderogabile necessità di restituire al lettore la complessità dell'esperienza umana ed esistenziale di quegli anni. La Resistenza raccontata da Verri «sono i corpi di coloro che l'hanno fatta e di coloro che sono morti per essa», scrive in una recensione al volume Racconti partigiani Demetrio Paolin, il legame insolubile tra chi si è liberato e chi è morto per la conquista della libertà.

Il 31 ottobre presso la libreria Feltrinelli di Parma si è svolto il terzo incontro: la presentazione del libro Qualcosa di Meglio. Biografia partigiana di Otello Palmieri di Alfredo Mignini ed Enrico Pontieri [Mignini e Pontieri 2019]. A dialogare con gli autori erano presenti Domenico Vitale e Paolo Capuzzo (Università di Bologna). La biografia di Palmieri è uno spaccato dalla società italiana del Novecento: Resistenza, emigrazione, militanza e disillusione politica sono i temi portanti. Il racconto - una via di mezzo tra intervista, saggio e romanzo storico vede i due autori e le proprie riflessioni come privilegiato punto d'osservazione. 
Un prezioso esempio di fonte orale che nella nostra rassegna ha permesso di porre il focus su una storia apparentemente marginale, di provincia e non conosciuta, che racchiude in sé però gli elementi determinanti di quella particolare stagione italiana.

Infine, l'evento di chiusura di questa prima edizione di Ottobre Partigiano si è tenuto il 5 novembre presso il Cinema Edison di Parma con la proiezione del film L'ultimo giorno di Amedeo Cavalca e Carlo Ugolotti. Prodotto e reso possibile dall'associazione Amici della Biblioteca San Leonardo in collaborazione con Isrec, il documentario racconta l'eccidio nazista che ha avuto luogo nella periferia agricola di Parma - nelle località di Pizzolese, Case Vecchie e Ravadese - il 25 aprile 1945. Il paradosso di un massacro che ha colpito piccole realtà contadine proprio nel giorno in cui "per convenzione" si celebra la fine del conflitto in Italia.

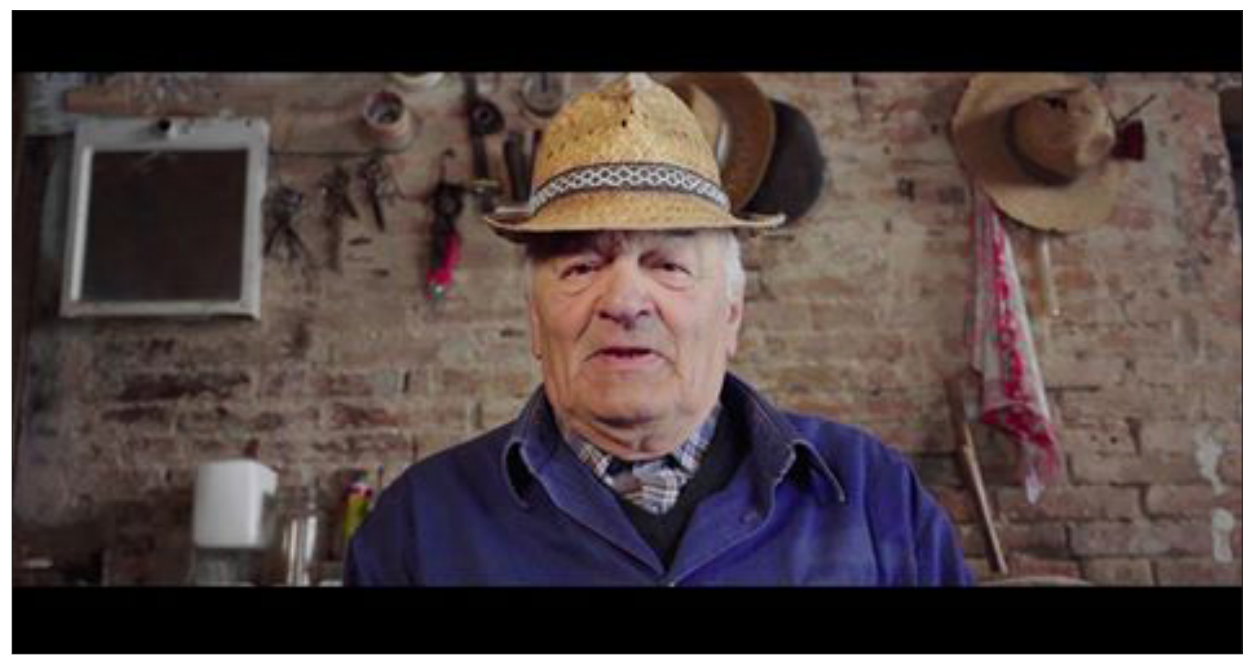

Fig. 6. Dal documentario "L'ultimo giorno sull'eccidio di Ravadese, Pizzolese e Case Vecchie"

Il documentario è costruito sul racconto a più voci dei testimoni-bambini di allora, una narrazione in cui si alternano, con ritmo incalzante e coinvolgente, interviste e frammenti di video dell'epoca. Il ruolo della memoria e il valore delle testimonianze dei sopravvissuti nella ricerca storica e nella narrazione filmica sono stati alcuni dei temi affrontati nel dibattito seguito alla proiezione, a cui hanno partecipato gli autori insieme a Marco Minardi e Mario Renosio (Istituto per la storia della Resistenza e della società contemporanea di Asti). L'evento era incluso nel cartellone del Parma Film Festival.

L'intero ciclo di incontri è stato accolto con interesse dalla cittadinanza, registrando ogni volta una buona partecipazione di pubblico di varie fasce d'età. L'apporto 
dell'accompagnamento musicale ha contribuito al successo delle serate proposte, così come la scelta di adottare una formula espositiva di tipo più divulgativo, caratterizzata dallo storytelling, che ha permesso di avvicinare un maggior numero di persone a temi spesso trattati in modo più tecnico e quindi meno fruibile. L'intento futuro è di organizzare altre edizioni mantenendo queste caratteristiche espositive, proponendo racconti e riflessioni su un fenomeno tanto importante, che merita di essere approfondito anche attraverso queste modalità più accessibili, che riescono a incuriosire e appassionare quanti non avevano mai pensato di partecipare prima a delle conferenze a tema storico.

\section{Bibliografia}

Badi F. 2015, Ines Bedeschi. Ritrovare un'assenza, Parma: Isrec Parma

Barbieri V. (ed.) 1975, La popolazione civile di Parma nella guerra 40-45, Parma: ANVCG

Berti G. 1975, Linee della Resistenza e liberazione piacentina, Piacenza: Isrec Piacenza

Casali L. e Gagliani D. (eds.) 2008, La politica del terrore. Stragi e violenze naziste e fasciste in Emilia Romagna, Napoli: L'Ancora del Mediterraneo

Fulvetti G. e Pezzino P. (eds.) 2016, Zone di guerra, geografie di sangue. L'Atlante delle stragi naziste e fasciste in Italia (1943-1945), Bologna: il Mulino

Gentile C. 2015, I crimini di guerra tedeschi in Italia, Torino: Einaudi

Gori G.M. e De Maria C. (eds.) 2020, Cinema e Resistenza, Roma: BraDypUS

Mignini A. e Pontieri E. 2019, Qualcosa di Meglio. Biografia partigiana di Otello Palmieri, Bologna: Pendragon

Tarantini L. 1978, La Resistenza armata nel parmense, Parma: Grafiche Step

Verri G. 2012, Partigiano inverno, Roma: Nutrimenti

Verri G. 2015, Racconti Partigiani, Pordenone: Edizioni Biblioteca dell'immagine

Verri G. 2019, Un altro candore, Roma: Nutrimenti 